\title{
Automated control system to monitor dielectric losses in polymers
}

\author{
Denis Butorin ${ }^{1, *}$ \\ ${ }^{1}$ Irkutsk State Transport University, 664074 Irkutsk, Russia
}

\begin{abstract}
The basic property of the processed polymers when it comes to high-frequency heat treatment is their dielectric losses. The purpose of the present study is elaboration of automated control system to monitor dielectric losses in polymers during heating. The technique developed in the given paper implies linear uniform heating of the studied material by contact method with the given velocity and when heating is off, periodic short-term action of high-frequency electric field on the polymer after each temperature growth of polymer per preset degree. The studies conducted in the automatic mode allowed obtaining new knowledge on changing electro-physical properties of polymers during heat treatment; and determining the new parameter to control polymer state while highfrequency processing; distinguishing and identifying different relaxation transitions of polymer materials during heating and thus broadening applicability of high-frequency heat treatment.
\end{abstract}

\section{Introduction}

High-frequency (HF) heat treatment is currently one of the most common among insignificant number of polymer heat treatment techniques (convective, contact, vacuum, infrared and compound); and it is considered as the most advanced, energy and resource saving processing technique of polymer components [1-4]. HF heat treatment technique is based on the polymer ability to dissipate energy in the electric field [2, 5-8]. Dielectric loss angle $\delta$ and its tangent $\operatorname{tg} \delta$ are used to describe this ability $[9,10]$.

Russian and foreign scientists $[1,2,5,6,11,12]$ made a significant progress in HF heat treatment technique development, particularly regarding diagnostics, drying, welding and recovery of the strength properties of polymer products. Furthermore, literature review indicates that control of HF treatment quality and relaxation state of polymer during treatment can be performed based on the single parameter of electric thermal equipment operation - anode current $I_{a}$ of HF generator connected analytically with $\operatorname{tg} \delta$. The research in $[1,2,6,12-14]$ uses this parameter $\left(I_{a}\right)$ as a controlled one. At this point it should be noted that due to few previous studies of HF influence on the polymer and the fact that polymer polarity was not considered, control of the polymer state in existing process procedures of HF treatment is attached just to the two relaxation transitions (maximum and

\footnotetext{
* Corresponding author: den butorin@mail.ru
} 
minimum $\left.I_{a}(T)\right)$. The temperature dependency $\operatorname{tg} \delta$ contains several extreme values divided into dipole-segmental losses $(\alpha)$ and dipole-group losses $(\beta, \gamma, \delta)[1,9,10]$.

To provide quality control of process procedures of polymers HF heat treatment it is required to develop a technique for controlling their dielectric losses described by the dependency $\operatorname{tg} \delta(T)$. This will allow establishing new controlled parameters of treatment, broadening the list of treated polymer materials, getting new knowledge on changing of their electro-physical parameters during treatment and widening applicability of HF heat treatment. Therefore, the present study aims to elaborate an automated technique to control dielectric losses in polymers during heating.

\section{Materials and methods}

To control dielectric losses in polymers a technique was developed which suggests linear uniform heating of the material under study by contact method with the velocity of $2{ }^{\circ} \mathrm{C} /$ min and periodic short-term ( 1 second) impact on the polymer, when heating is off, by $\mathrm{HF}$ electric field (voltage $U_{p}=4000 \mathrm{~W}$ ) after each temperature growth of polymer specimen by $2{ }^{\circ} \mathrm{C}$.

The technique was implemented on the improved and automated experimental setup ASNI VC (automated system for research studies of high-frequency treatment) [1, 6, 1214]. Hardware component of the improved setup differed from the existing setup by exception of the press influence on heating and deformations of the specimen under study. Press unit (force $0 \ldots 400 \mathrm{~kg}$ ) was turned off, while the weight $P$ of upper moving plate of operating capacitor was taken up by elastic members up to $P \geq F_{T P o n}$, where $F_{T P o n}$ - friction force in supports. The final scheme of experimental setup is shown in Figure 1.

In simplified form the developed technique includes the following operations. The specimen from polymer material 1 is placed between electrodes 3 of a parallel plate operating capacitor connected to a HF generator 4, while a thermal-electrical insulator 2 is placed between the studied specimen 1 and electrode 3 . The lower electrode as a whole is an electrical heating element 6 , providing heating of the specimen up to melting temperature $T_{m}$ (determined experimentally) with the velocity of $2{ }^{\circ} \mathrm{C} / \mathrm{min}$. Device 5 has a hole with inserted heat and electrical insulation layer and a thermocouple 7 to control surface temperature of the studied specimen 1. Anode current on the operating capacitor is measured automatically using the current sensor 8-1 after switch-on of the HF generator. Specimen temperature compared to the existing ASNI VC [1, 6, 12-14], is controlled using the thermocouple 7-1 only when HF generator is switched off, thus, measuring of temperature and current $I_{a}$ are synchronized automatically in phase opposition. Output signals from the thermocouple 7-1 and current sensor 8-1 enter a computing unit 10, given as micro controller, connected to the personal computer via COM-port. Computing unit 10 includes the following sub-units:

- temperature control sub-unit 6-2;

- anode current control sub-unit 8-2;

- linear thermal expansion control sub-unit 11-2. 


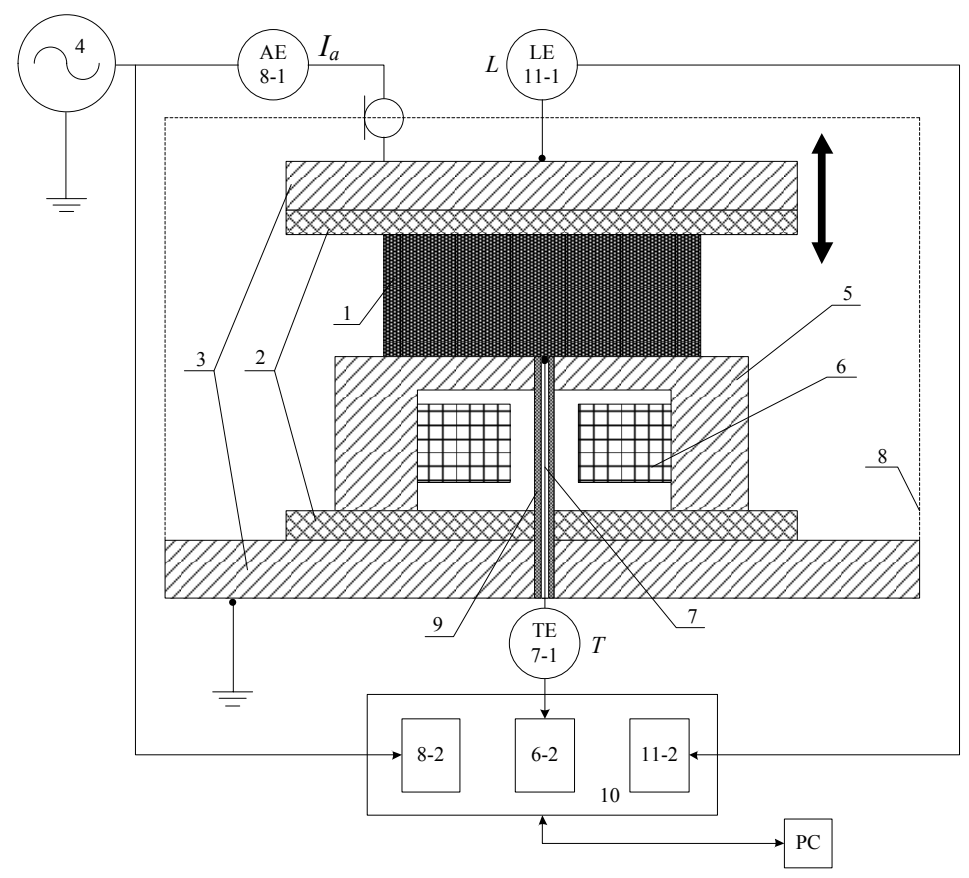

Fig. 1. Scheme of automated experimental setup to define amperometric dependency of dielectric losses in polymer materials, where 1 is polymer specimen under study; 2, 9 are thermal-electrical insulators; 3 is electrodes of operating capacitor; 4 is HF generator; 5 is a device for specimen heating; 6 is electrical heating element; $6-2$ is temperature control sub-unit; 7 is a thermocouple; $7-1$ is a thermocouple-temperature gauge; 8 is a protective screen; $8-1$ is a current sensor; $8-2$ is an anode current control sub-unit; 10 is a computing unit; $11-1$ is a linear thermal expansion sensor; $11-2$ is a linear thermal expansion control subunit.

To record the moment when the specimen reaches melting temperature, Balluff Micropulse BIW inductive linear position sensor with analog output signal $0 \ldots+10 \mathrm{~W}$ was attached to the mechanism of upper moving plate of the operating capacitor; the data was received by the computing unit 10 . The software component of ASNI VC was improved by the developed algorithm for controlling the research process. Its flow-diagram is shown in Figure 2. 


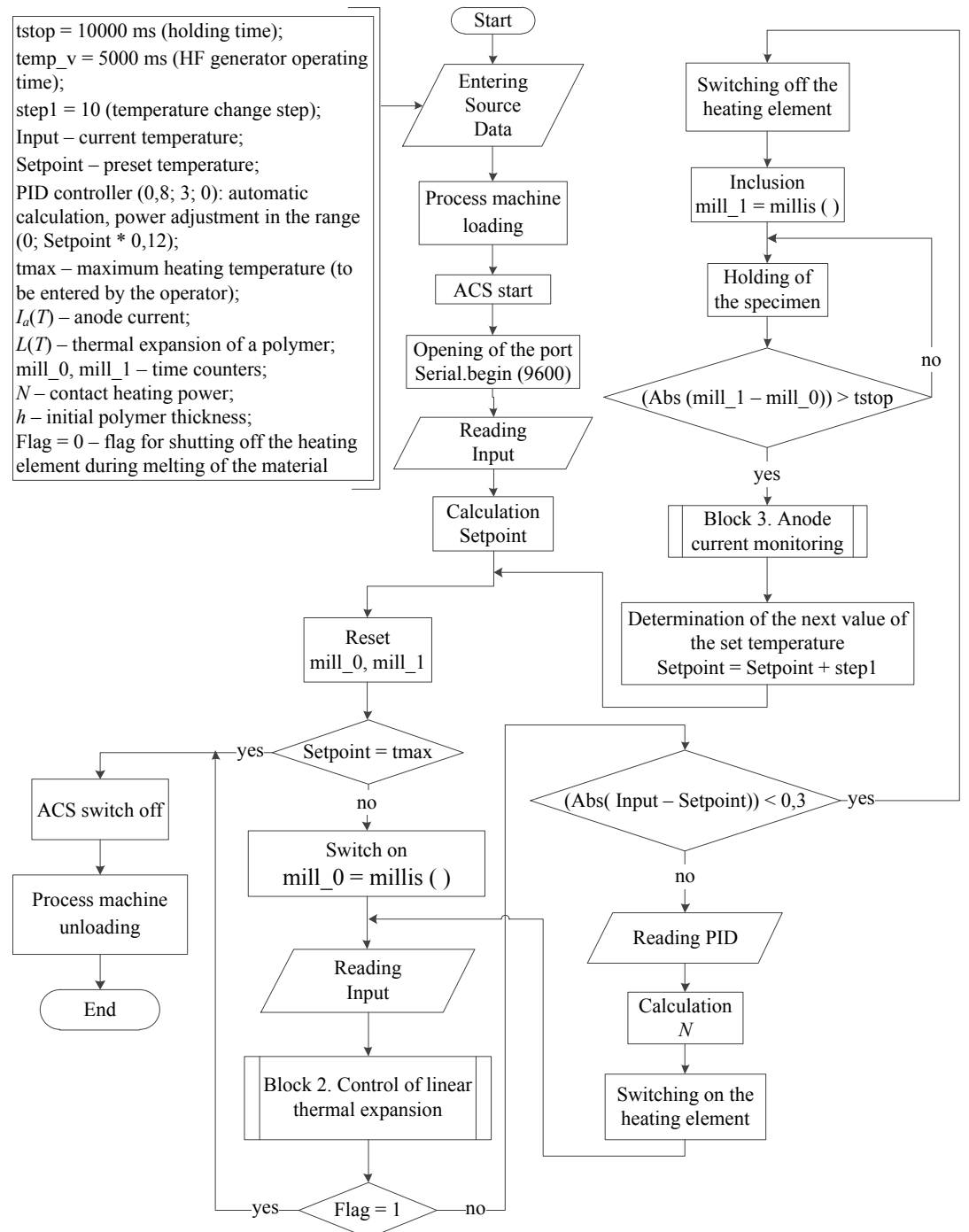

Fig. 2. Flow-diagram of the algorithm for controlling the process of linear homogenous heating of material under study and periodic short-term action of HF electric field when heating is off.

The elaborated control algorithm consists of three units:

- unit 1 of uniform heating of the material under study (basic unit);

- unit 2 of linear thermal expansion control during automated research (Fig. 3, a);

- unit 3 of anode current control (Fig. 3, b).

The given algorithm takes surface temperature of polymer specimen $T_{\text {surf }}$, its linear thermal expansion $L$ and anode current of HF generator as the controlled parameters; while contact heating capacity $N$ was accepted as a parameter-driven.

Resulting from the value of $I_{a}$ of $\mathrm{HF}$ generator the studies were conducted on dielectric losses of some strong-polar polymer materials (PVC-plasticate OMB-60) and polar polymer materials (polyamide PA6) according to the elaborated technique on the improved experimental setup of brand UZP 2500 with operating capacity of HF generator 27.12 MHz. The specimens' sizes were chosen due to the criteria of maximum efficiency 
treatment on HF setup and were presented as round discs $60 \mathrm{~mm}$ in diameter and $4 \mathrm{~mm}$ thick. 7 tests were conducted on each of the materials to get valid results.

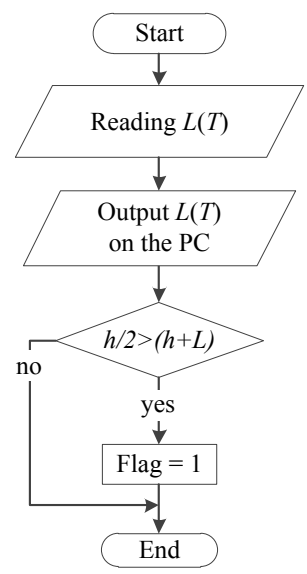

a

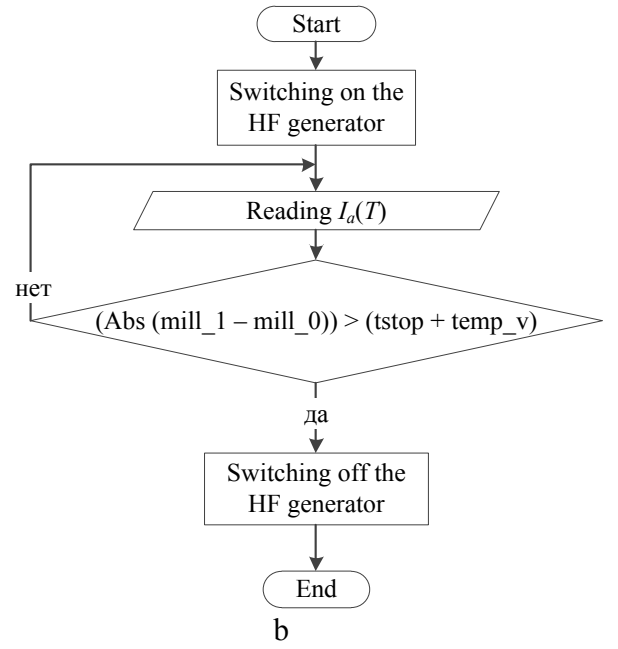

Fig. 3. Flow-diagram of sub-units: a - unit 2 linear thermal expansion control; $b$ - unit 3 anode current control.

\section{Experiment and results}

Resulting from the obtained experimental data dependency graphs between $I_{a}(T)$ and $L(T)$ were built for the given polymer materials (Fig. 4). Analysis of the given dependencies $I_{a}(T)$ and $L(T)$ provides interesting data. The dependency graph $I_{a}(T)$ (Fig. 4, a), obtained while polyamide treatment shows two extreme values (maximum and minimum points of $I_{a}$ ), while during PVC treatment $I_{a}$ extreme values were not observed (Fig. 4, b).

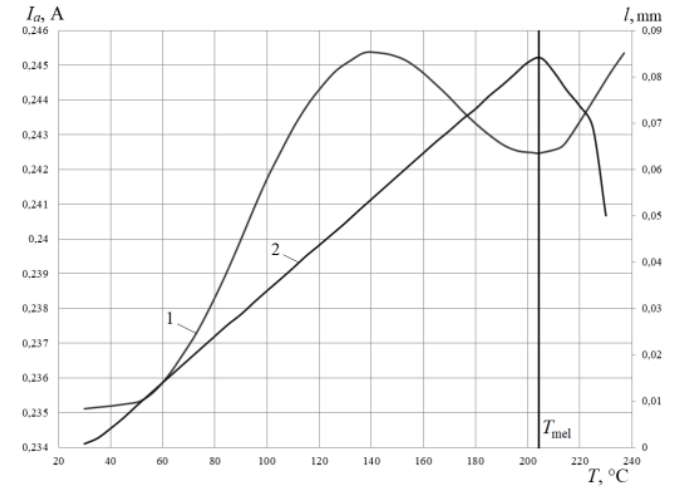

a

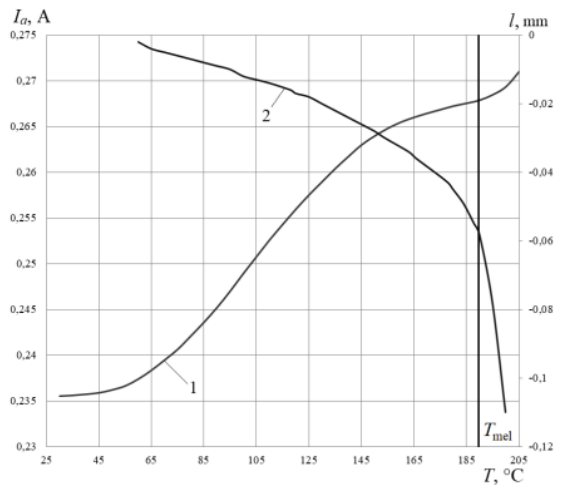

b

Fig. 4. Dependency graphs of $I_{a}(T)(1)$ and $L(T)$ (2) obtained during treatment of polyamide PA6 (a) and PVC-plasticate OMB-60 (b).

Combining the dependency graphs of $I_{a}(T)$ and $L(T)$ obtained during polyamide treatment (Fig. 4, a) enables to identify the minimum of $I_{a}$ as the moment when polymer reaches the melting temperature, its temperature value corresponds to the reference data. 
Maximum of $I_{a}(T)$, as described in literature sources reflects the state when polymer reaches high-elastic state (dipole-segmental losses).

It also should be noted that for certain polymers the curve $L(T)$ has monotonic dropdown nature (PVC-plasticate), for other materials (polyamide PA6) it looks like a curve with a bending point. It is obvious that this fact depends on materials properties which vary during heating process, thus, it can be used for polymer identification.

Supported by previous studies [1] and analyzing the dynamics of anode current change the velocity of anode current change $d I_{a} / d T$ was defined using differential operator of left finite difference of the first approximation order. The graph showing the velocity of anode current change $d I_{a} / d T$ for the mentioned materials is given in Figure 5.

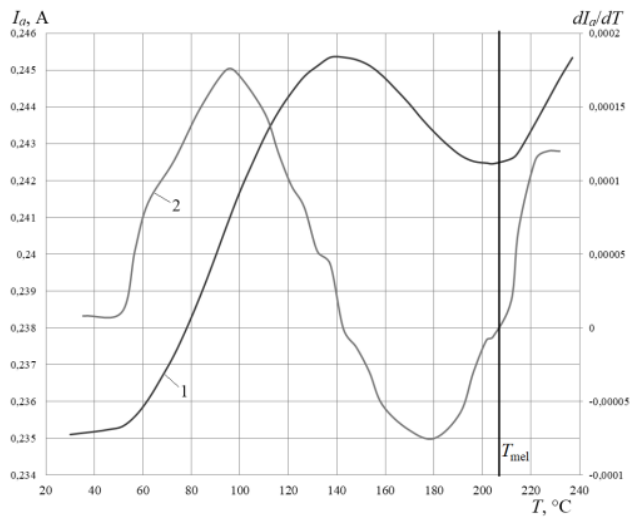

a

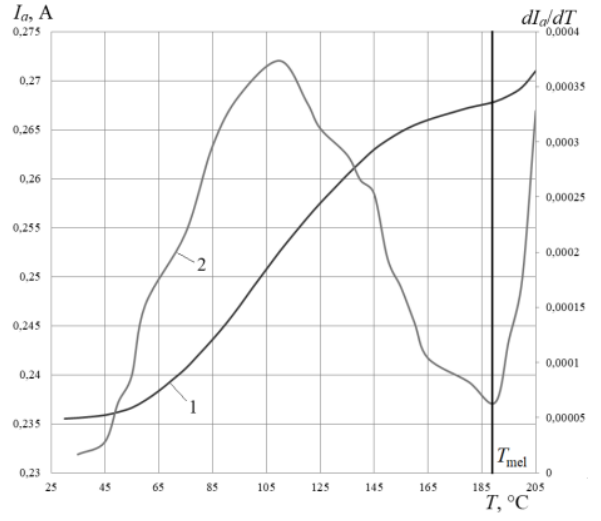

b

Fig. 5. Dependency graphs of $I_{a}(T)(1)$ and $d I_{a} / d T(2)$ obtained during treatment of polyamide PA6 (a) and PVC-plasticate OMB-60 (b).

Data analysis $\left(d I_{a} / d T\right)$ revealed that the velocity of anode current change can be also used as the controlled parameter of treatment. During processes of HF treatment of polar polymers, such as polyamide PA6 (Fig. 5, a), as compared to strong-polar polymers (PVCplasticate OMB-60), their melting state (minimum $I_{a}$ ) or dipole-segmental mobility (maximum $I_{a}$ ) can be defined by $d I_{a} / d T$ passing through zero and reaching first the negative value, and then the positive value.

During HF-treatment of PVC-plasticate (Fig. 5, b), which shows no extreme values $I_{a}$, calculation of $d I_{a} / d T$ reveals new reference points (extreme values $d I_{a} / d T$ ), and along with them new opportunities to organize process procedures of $\mathrm{HF}$ heat treatment and automation of their control. Minimum $d I_{a} / d T$ during treatment of PVC-plasticate was first identified in this study as the moment when plasticate reaches the melting state. Maximum $d I_{a} / d T$ obtained during treatment of both studied polymers was identified as reaching the state of dipole-group losses $\beta(\operatorname{tg} \delta)$.

\section{Conclusion}

The present study allowed developing and automating the technique to control dielectric losses in polymers through anode current of HF generator. In the course of experimental data analysis the new data was obtained on changing electrical and physical properties of polymers during heat treatment. The new controlled parameter in HF treatment was established, i.e. the velocity of anode current change $d I_{a} / d T$.

The developed technique enabled to determine and identify different relaxation transitions of polymer materials during heating along the extreme values $I_{a}$ and $d I_{a} / d T$, as 
well as the dependencies $L(T)$. From the viewpoint of extremal systems controlling the process of polymer HF treatment each relaxation transition possesses practical importance. Thus, the maximum state $I_{a}$ corresponds to the effective temperature of drying and recovering the strength properties of products from polar polymers. The minimum state $I_{a}$ corresponds to the temperature of welding and adhesive bonding processes during treatment of polar polymers; while the minimum state $d I_{a} / d T$ refers to treatment of strong-polar polymers. The maximum $d I_{a} / d T$ can be applied as the reference point while impregnating polymer with various substances, e.g. motor oils. All this broadens the application field of high-frequency heat treatment.

Further research will be focused on distinguishing new classification of polymer materials considering their controllability when building extremal systems and elaboration of automated control systems of high-frequency treatment processes.

\section{References}

1. A.V. Livshits, Doctoral thesis, Irkutsk State Transport University, Irkutsk (2016). (in Russian)

2. N.G. Filippenko, Thesis of Candidate of technical sciences, Irkutsk State Transport University, Irkutsk (2012). (in Russian)

3. Yu.S. Arkhangelskiy, Vestnik of Saratov State Technical University, 4, Issue 3, 5-15 (2011). (in Russian)

4. A.V. Markov, Yu.P. Yulenets, Elektrotehnika [Russian Electrical Engineering] 7, 60 64 (2007). (in Russian)

5. S.N. Rumynskiy, Thesis of Candidate of technical sciences, Saint-Petersburg State Institute of Technology, Saint-Petersburg (2005). (in Russian)

6. A.G. Larchenko, Thesis of Candidate of technical sciences, Irkutsk State Transport University, Irkutsk (2014). (in Russian)

7. T. Ohe, Y. Yoshimura, Sen'i Gakkaishi, 1(65), 64-70 (2009).

8. W.R. Tinga, S.O. Nilson, The Journal of microwave power, 1, 27-33 (1973).

9. V.K. Kryzhanovskiy, V.V. Burlov, A.D. Panimatchenko, Yu.V. Kryzhanovskaya, Tehnicheskie svojstva polimernyh materialov [Technical properties of polymeric materials] (Professiya, St-Petersburg, 2005). (in Russian)

10. A.A. Tager, Fiziko-himija polimerov [Physicochemistry of polymers] (Nauchnyj mir, Moscow, 2007). (in Russian)

11. A.V. Livshits, JP Journal of Heat and Mass Transfer 14(2), 219-226 (2017).

12. S.I. Popov, Thesis of Candidate of technical sciences, Irkutsk State Transport University, Irkutsk (2013). (in Russian)

13. A.V. Livshits, Proceedings of the International Scientific Conference "Science and practice: new discoveries», 106-114 (Czech Republic, Karlovy Vary - Russia, Moscow, 2015). (in Russian)

14. A.V. Livshits, Engineering and Automation Problems, 4, 54-60 (2015). (in Russian) 